\title{
Um Estudo de Caso Exploratório sobre a Internalização de Conceitos sobre Eletrostática: A influência da Hipercultura e Mediação Digital
}

\author{
Juliano Rodrigues Rocha PPGECIM - ULBRA juliano.rrocha@ hotmail.com \\ Agostinho Serrano de Andrade Neto PPGECIM - ULBRA asandraden@gmail.com
}

\begin{abstract}
Resumo
O presente trabalho busca identificar o impacto das tecnologias de informação em termos das mudanças cognitivas resultantes de seu uso, dentro do ensino de Física. Para esse fim trabalhamos sob a luz de uma teoria cognitiva baseada na ideia de que a inteligência humana não resulta apenas do funcionamento cerebral, mas também da complementação desse funcionamento pelo processamento auxiliar realizado por estruturas externas ao individuo, a Teoria da Mediação Cognitiva (TMC). Como foco da investigação se tomou a compreensão do conceito de campo elétrico, o grupo observado foi submetido a métodos de analise e uma intervenção com a utilização de simuladores computacionais. Os resultados são expressivos e apontam para uma real mudança conceitual a respeito da teoria de campo elétrico devido à utilização de simuladores.
\end{abstract}

Palavras Chave: Simulações Computacionais, Teoria de Campos Conceituais, Mediação Cognitiva, Hipercultura.

\section{An exploratory case study of the internalization of electrostatics concepts: The influence of the hyperculture and digital mediation}

\begin{abstract}
This study aims to identify the impact of information technologies in terms of cognitive changes resulting from this process, in the field of Physics Education. To this end we work under the light of a cognitive theory based on the idea that human intelligence derives not only from brain function, but also the completion of this operation by the auxiliary processing done by external structures to the individual, the Theory of Cognitive Mediation (TMC). As a focus of research became the understanding of the concept of the electric field, the observed group was subjected to methods of analysis and intervention with the use of computer simulators. The results are expressive and show a real conceptual on the theory of the electric field due to the use of simulators.
\end{abstract}

Keywords: Computer Simulations, Conceptual Fields Theory, Cognitive Mediation, Hyperculture.

\section{Introdução}

A visualização desempenha um importante papel na compreensão de conceitos científicos devido ao grande número de representações utilizadas como forma de expressá-los. A Física é uma ciência particularmente visual e a utilização de representações como forma de expressão de conceitos e procedimentos físicos é realizada de forma ampla. Essa característica gera no aluno a necessidade da construção de modelos mentais e esquemas de assimilação. A tarefa de ensino do conceito de campo, em especial campo elétrico, tem sido sobremaneira difícil. Aparecem complicações que se devem às várias razões, explanadas em várias publicações na literatura de ensino de ciências. Algumas delas são destacadas por Martin e Solbes (2001): 
- A dificuldade que encontramos para realizar uma introdução qualitativa do campo, dado o nível de abstração dos conceitos implicados.

- Não podemos relacionar facilmente com a experiência cotidiana dos alunos, como pode ocorrer com os conceitos de mecânica.

- Durante o processo de ensino ocorre frequentemente que as interações entre partículas podem ser descritas de diversas formas (forças, campos, energias, etc.) e podemos desorientar os alunos se não ficar suficientemente claros os conceitos, estabelecendo suas relações, suas diferenças e âmbitos de aplicação.

Nesse trabalho, tomamos a hipótese que observações sistemáticas de gestos de estudantes podem não só auxiliar nessa análise como preencher as lacunas nas expressões verbais, podendo também oferecer informações valiosas sobre as ideias dos alunos, Esses gestos estão fortemente ligados ao conceito de representações de Gerard Vergnaud, externalizando os invariantes operatórios que esse estudante já havia adquirido.

Este trabalho tem como objetivo apresentar evidências de como o uso de softwares de simulação no ensino de Física, mais especificamente em eletrostática, pode se tornar uma ferramenta de processamento extra cerebral, e quais as possíveis modificações que ocorrem na estrutura cognitiva dos estudantes nesse processo.

Utilizaremos como principal referencial epistemológico a Teoria dos Campos Conceituais de Vergnaud (TCC), associada à Teoria da Mediação Cognitiva em Rede (TMC), por sua abordagem em relação à mediação por computador, já que o foco do nosso trabalho de pesquisa está no estudo de como ocorre a aprendizagem de conceitos físicos com a mediação por computador, em especial pelo uso de softwares de simulação de campo elétrico. Outro aporte teórico utilizado de forma complementar é a Teoria da Carga Cognitiva (SWELLER, 1988), que considera, também de forma resumida, que temos uma memória de trabalho finita e que qualquer processo que permita a automatização de esquemas utilizados no aprendizado de algum conceito ou processo, "libera" memória de trabalho para que se possa utilizar em outras tarefas cognitivas, na direção do aprendizado.

\section{Teoria de Campos Conceituais de Vergnaud (TCC)}

Esta teoria diz que para estudar e compreender como os conceitos evoluem na mente de um sujeito, por meio de suas experiências, é preciso considerar o conceito (C) como uma terna de conjuntos, ou seja, $\mathrm{C}=(\mathrm{S}, \mathrm{I}, \mathrm{R})$, onde:

- S é o conjunto de situações que dão significado e utilidade ao conceito;

- I é o conjunto de invariantes operatórios associados ao conceito (objetos, propriedades e relações), que podem ser reconhecidas e usadas pelos sujeitos, de forma a analisar e dominar aquelas situações;

- $\mathbf{R}$ é o conjunto de significantes, isto é, a representação simbólica, linguística, gráfica ou gestual, que podem ser utilizadas para representar aqueles invariantes, e, desta forma, representar as situações e os procedimentos para lidar com eles.

Em termos psicológicos, o autor explicita que $S$, o referente, é a realidade; e I e $R$ representam os dois aspectos integrantes do pensamento, o significado (I) e o significante $(\mathrm{R})$.

É fundamental considerar esses três conjuntos simultaneamente - situações, invariantes operatórios e representações simbólicas - ao longo da aprendizagem, para estudar o desenvolvimento e o uso de um conceito (VERGNAUD, 1981). 


\title{
Teoria da Mediação Cognitiva em Rede (TMC)
}

Em um artigo publicado na revista Computers in Human Bahavior, Souza et al. (2004) procura explicar o processamento da informação pelo cérebro, propiciando uma abordagem ampla para a cognição humana. A TMC é uma teoria contextualista e construtivista. Os autores apresentam o desafio de "fornecer uma síntese teórica coerente de teorias psicológicas e estruturais que são geralmente vistas como separadas, ou mesmo em conflito umas com as outras, de modo a produzir um modelo unificado"(SOUZA et al., 2004, pg 2321).

A TMC é fundamentada e referenciada em cinco premissas relativas à cognição humana e ao processamento de dados:

\begin{abstract}
(...) 1) A espécie humana tem como maior vantagem evolutiva a capacidade de gerar, armazenar, recuperar, manipular e aplicar o conhecimento de várias maneiras; 2) A cognição humana é efetivamente o resultado de algum tipo de processamento de informação; 3) Sozinho, o cérebro humano constitui um finito e, em última instância, seu recurso de processamento de informação é limitado; 4) Praticamente qualquer sistema físico organizado é capaz de executar operações lógicas em algum grau; 5) Seres humanos complementam o processamento da informação cerebral por interação com os sistemas físicos externos organizados. (SOUZA et al., 2004, p.2321, tradução nossa).
\end{abstract}

A capacidade do cérebro humano de complementar o processamento de informações com o uso de sistemas físicos organizados é, para os autores desta contribuição, uma das suas melhores características, e culmina com a invenção dos computadores. Com o surgimento da Revolução Digital, houve mudanças importantes nas sociedades e culturas de todo o mundo, influenciando o homem em níveis individuais e coletivos pelo impacto das tecnologias digitais sobre o pensamento, surgindo desse contexto uma nova cultura, a hipercultura (SOUZA et al., 2004).

Os autores salientam também que mesmo antes dos adventos computacionais o cérebro humano já se utilizava de mediações externas auxiliares, porem essas eram mediações físicas do ambiente, objetos, sistemas simbólicos e artefatos. Essa mediação, para os autores, era denominada de Mediação psicofísica cultural, um exemplo dessa mediação é utilizar as formas criados por limalha de ferro quando expostas a um campo magnético para descrever campo elétrico.

A cognição humana é o resultado de processamento de informações e uma parte importante desse processamento é realizado fora do cérebro. Nesse sentido, utilizamos o processamento externo através da interação com estruturas do ambiente para aumentar a capacidade de processamento de informações feitas pelo cérebro. Por exemplo, quando utilizamos um computador para processar informações, ou mesmo realizar um cálculo mais complexo, estamos utilizando-o como um mecanismo externo de mediação. Para tanto, precisamos construir alguns mecanismos internos que nos possibilite manusear este computador e compreender não somente o seu processamento, mas também as informações que ele está nos oferecendo.

Estes mecanismos internos é que tornam possível a utilização dos mecanismos externos e são chamados pelos autores de drivers, tecendo uma analogia à computação, própria a uma abordagem baseada na metáfora computador-cérebro da psicologia cognitiva.

\section{Os Drivers}

A TMC interpreta driver como dispositivos que trabalham como "máquinas virtuais" internas, que possuem um papel importante na definição do pensamento humano no contexto da mediação e vão para além da "conexão" com o mecanismo externo: 
É razoável supor que os mecanismos internos de mediação funcionem através da produção de um shell, ou seja, de uma "máquina virtual" que "espelha" ou "representa" o mecanismo externo. Trata-se de um processo necessário para o estabelecimento de uma interface entre o cérebro e o mecanismo extra cerebral, mas também permite, até certo ponto, uma "emulação" ao menos parcial dos mecanismos externos em questão. Isso implica, portanto, numa internalização parcial dos mecanismos externos, o que ajuda a explicar por que as habilidades permanecem aumentadas mesmo quando os mecanismos externos estão ausentes. (SOUZA, 2004, p. 81-82)

Isto significa que, para garantir o processo de mediação cognitiva com um mecanismo externo, nosso cérebro cria competências específicas para se comunicar com este mecanismo - que é um auxiliar no processamento de informação - e, a partir dessa mediação, adquire um ganho de processamento de informações que se mantém mesmo que a conexão com o mecanismo externo seja interrompida. E esse ganho de processamento de informações é considerado pelo autor como aquisição de conhecimentos. Souza et al. (2004) resume este processo da seguinte forma:

A fim de integrar o processamento de informação feito pelo cérebro com um executado por mecanismos externos, é necessário que haja uma ligação lógica entre estes dispositivos. Por outras palavras, alguma forma de traduzir entradas, saídas e processamento entre eles. Isto é muito semelhante ao ter de instalar "controladores de dispositivos" de software num sistema de computador, de modo que este seja capaz de reconhecer e fazer funcionar um equipamento externo específico, tal como uma impressora, um scanner ou outro dispositivo de armazenamento. Nos seres humanos, isto pode ser conseguido por meio de uma representação mental de um sistema físico que é constituído de um conjunto de "teoremas-em-ação", no sentido estabelecido pela teoria dos campos conceituais de Vergnaud (Vergnaud, 1997), que são análogos ao funcionamento dinâmico do referido mecanismo externo, por conseguinte, tornando possível a um indivíduo interagir com este mecanismo externo para fins de processamento de informação. Como tal, o desenvolvimento deste "mecanismo interno" ocorre por meio da interação entre o indivíduo e o sistema físico correspondente, isto é, através do processo descrito na Epistemologia Genética de Piaget como "equilibração". (SOUZA et al., 2012, p. 2321-2322, tradução nossa)

O mecanismo externo de processamento de informações - computador - interage com a estrutura cognitiva dos estudantes por mediação em função de que há a internalização de um conjunto de sistemas de signos (VYGOTSKY, 1984) capazes de fazer com que o estudante compreenda e internalize, mesmo que em parte, a informação que está sendo apresentada na tela. Esta contribuição vygotskiana é incorporada na TMC.

\section{Definição do Problema e Método de Pesquisa}

Tendo esse trabalho como objetivo apresentar evidências de como o uso de softwares de simulação no ensino de Física pode se tornar uma ferramenta de processamento extra cerebral, e quais as possíveis modificações que ocorrem na estrutura cognitiva dos estudantes nesse processo. Para tanto, utilizaremos os gestos descritivos e o vínculo desses gestos com o crescimento conceitual do estudante após a utilização de um software de simulação de campo elétrico, como compreendido pela TCC e TMC, acima descritas.

A atividade de pesquisa aplicada a uma amostra formada por estudantes de cursos de graduação, em uma universidade privada de Canoas, RS - Brasil, se constituía de 13 alunos, sendo 7 do grupo experimental e 6 do grupo controle. Esta atividade contou com cinco etapas: 
Etapa 1: Na primeira etapa um pré-teste ${ }^{1}$ de resolução de problemas que foi realizado com todos os alunos da amostra, antes de qualquer contato com o software. Cinco situações-problemas, típicas de livro-texto, foram propostas referentes a campo elétrico e interações entre cargas elétricas, respondidas livremente pelos alunos em base dos seus próprios conceitos, individualmente.

Etapa 2: Entrevista do pré-teste. A entrevista do pré-teste bem como as etapas seguintes foram aplicadas a uma parcela de 7 alunos da amostra, sendo 4 do grupo experimental e 3 do grupo controle, para a realização das entrevistas, foi utilizado o protocolo "think aloud" (SCHERR, 2008; STEPHENS \& CLEMENT, 2010). Essa técnica utiliza um método de coleta de dados em que, basicamente, o entrevistador e o entrevistado mantenham constante diálogo a respeito do que o entrevistado está pensando durante a execução de uma tarefa. As entrevistas foram gravadas com posterior transcrição e a análise das entrevistas foi realizada por análise gestual (MONAGHAN; CLEMENT, 1999). Esta metodologia de análise gestual já foi utilizada em outros trabalhos e se baseia no fato de que existe um vínculo estabelecido entre gestos descritivos e imagens mentais (STEPHENS \& CLEMENT, 2010).

Etapa 3: Nesta etapa, os estudantes do grupo experimental com o auxilio de um guia de simulação ${ }^{2}$ foram instruídos de como utilizar o software de simulação online "Taxas e Campos" "3e forma individual. Durante esta etapa, é necessário que os estudantes manipulem livremente o software, pois é nela que acreditamos ocorrer a internalização dos drivers inerentes às representações computacionais.

O software (Figura 1) permite que o usuário explore uma série de simulações de um sistema carga-campo elétrico.

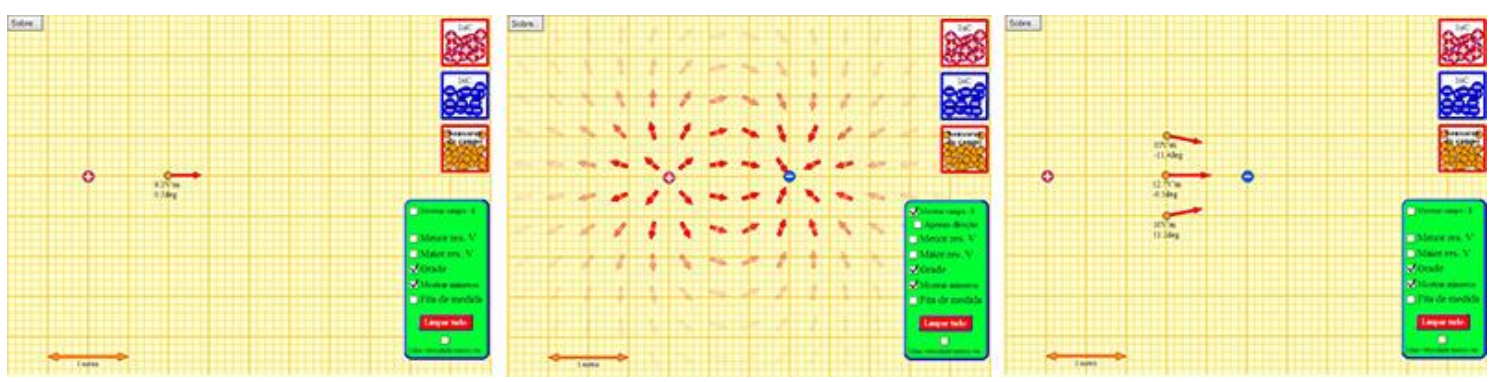

Figura 1 - Simulador utilizado no experimento.

Etapa 4: Posteriormente, após o uso do software, foi aplicado o pós-teste ${ }^{4}$, onde sem o auxílio do simulador, os estudantes efetuaram cinco situações-problemas propostas, referentes a campo elétrico e interações entre cargas elétricas.

Etapa 5: Entrevista do pós-teste, na entrevista do pós-teste se utilizou a mesma metodologia adotada na entrevista do pré-teste (etapa 2) seguindo os mesmos protocolos.

Posteriormente se fez o cruzamento dos resultados dos instrumentos entre as etapas bem como entre grupos e análise das representações captadas por vídeo. Cada representação captada em vídeo é apenas considerada uma representação descritiva se, em algum momento, o estudante denomina explicitamente ou implicitamente esta. 


\section{Analise dos Resultados}

Para exemplificar a aplicabilidade das teorias apresentamos os resultados desse estudo exploratório. Antes da utilização do software de simulação podemos observar, na entrevista do pré-teste, um maior número de eventos nos quais gestos descritivos são produzidos sendo esses indícios documentados da geração de imagens mentais (STEPHENS \& CLEMENT, 2010). Estas imagens mentais, por sua vez, constituem-se basicamente de representações e de invariantes operatórios implícitos no estudante. Com a analise do discurso verbal e do discurso não verbal do estudante se percebe os drivers internalizados por ele, podemos dizer que os drivers são dependentes do tipo de mediação onde a situação problema foi resolvida, ou seja, como antes do contato com o software o estudante utilizava formas de mediação psicofísicas e culturais, seus drivers eram psicofísicos e culturais.

Podemos verificar que o estudante "EZ" se utiliza de recursos psicofísicos para descrever uma situação problema, que corresponde a um driver de visualização. Nessa descrição o estudante utiliza de forma explicita uma representação externa para a resolução da situação problema, o estudante se apropria dessa representação tanto no discurso verbal quanto no não verbal. Quando o estudante tenta descrever o campo elétrico gerado por uma carga positiva, ele descreve o movimento de papeis sobre um gerador de Van der Graaff e em seu discurso não verbal, abre e fecha as mãos repetidamente simulando um movimento de repulsão, (Figura 2).

Estudante EZ: Não sei se da para comparar com aquela... Van der Graaff, não sei por que quando a gente fez o teste colocou os papeizinhos em cima, quando ligou expeliu os papeis [abre e fecha as mãos repetidamente], imaginei assim...

Até onde essa carga atingir o papelzinho vai [faz um movimento de afastamento em relação a um ponto imaginário], assim que perdeu o alcance da carga ele teria a questão da gravidade, foi isso que eu entendi.
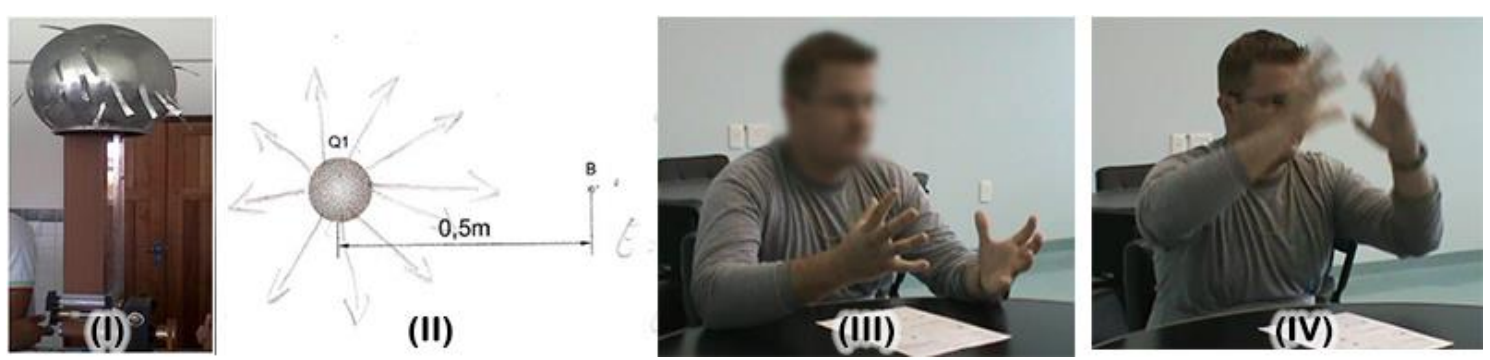

Figura 2 - (I) Gerador de Van der Graaff com papeizinhos colados, (II) representação desenhada por EZ de campo elétrico, (III, IV) gestos descritivos associados.

A análise do pós-teste em conjunto com a entrevista do pós-teste evidência uma clara mudança conceitual por parte dos estudantes do grupo experimental. Enquanto os alunos do grupo controle continuam com drivers psicofísicos culturais os estudantes do grupo experimental, que foram submetidos à intervenção do software, evidencia uma mudança cognitiva devido a esse processo, caracterizada pela aquisição de drivers hiperculturais.

A evolução conceitual do estudante "EZ" corrobora essa evidência, ao analisar as imagens de vídeo, em conjunto com as respostas dos pré-testes e pós-testes, podemos inferir, à luz do marco teórico, que os estudantes, ao interagirem com o software de simulação (representada por um mecanismo externo), desenvolveram novos drivers hiperculturais. Estes drivers se incorporaram na estrutura cognitiva dos estudantes, 
conferindo-lhes a possibilidade de avançar em termos de pensamento lógico, que é sustentada por manifestações dos estudantes no pós-teste, representado na (Figura 3) e (Figura 4) referentes a evolução cognitiva do estudante "EZ". No caso, todas as tarefas aqui reportadas solicitavam que o estudante desenhasse o vetor campo elétrico resultante especificamente no ponto $\mathrm{A},(\mathrm{B}$ e $\mathrm{C})$ :

Estudante EZ: Naquele exercício que a gente fez no computador, eu entendi melhor como funcionado o campo de força... o campo elétrico né, ai eu imaginei aquilo lá, então o vetor o campo elétrico mesmo foi referente depois do $\mathrm{B}$, tipo assim se tá pequena a força mas tem força vai ser em relação ao ponto $\mathrm{B}$, então eu entendi que não esta perguntando desse meio aqui, esta perguntando referente ao ponto B então daqui vai sair uma força ou positiva ou negativa [com o dedo descreve no papel o vetor], no caso como aqui a carga é positiva então foi do ponto B e tá indo para lá. [faz movimento de afastamento em relação a um ponto imaginário]

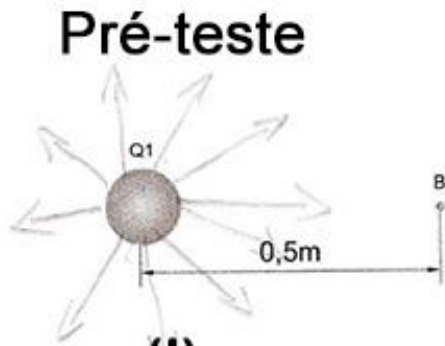

(I)
Simulador

(Intervenção)

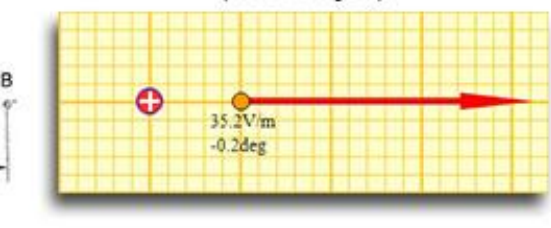

(II)

\section{Pós-teste}

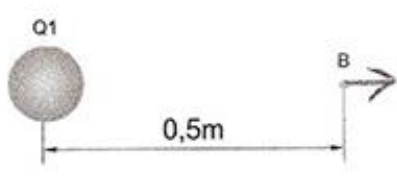

(III)

Figura 3 -(I) Representação desenhada por EZ de vetor campo elétrico, (II) imagem capturada da simulação, (III) representação desenhada por EZ de vetor campo elétrico

Estudante EZ: (...) ai para desenhar o que eu imaginei aquele exercício que a gente fez no computador, então aqui esta mais forte, esquecendo isso aqui se fosse só usar isso aqui ela ficaria numa linha reta o campo de força, se fosse esquecer essa aqui ela ficaria para cá numa linha reta se as duas fossem da mesma força né [com o dedo descreve linhas no papel], vamos supor os dois fossem $1 \mathrm{nC}$ ou os dois fossem $2 \mathrm{nC}$ então as duas estariam com a mesma força iam se encontrar aqui e essa flecha ficaria reta, mas como essa aqui tem praticamente o triplo então essa aqui tem mais força então a flecha vai ficar um pouco inclinada para cima. [faz movimento obliquo para cima]
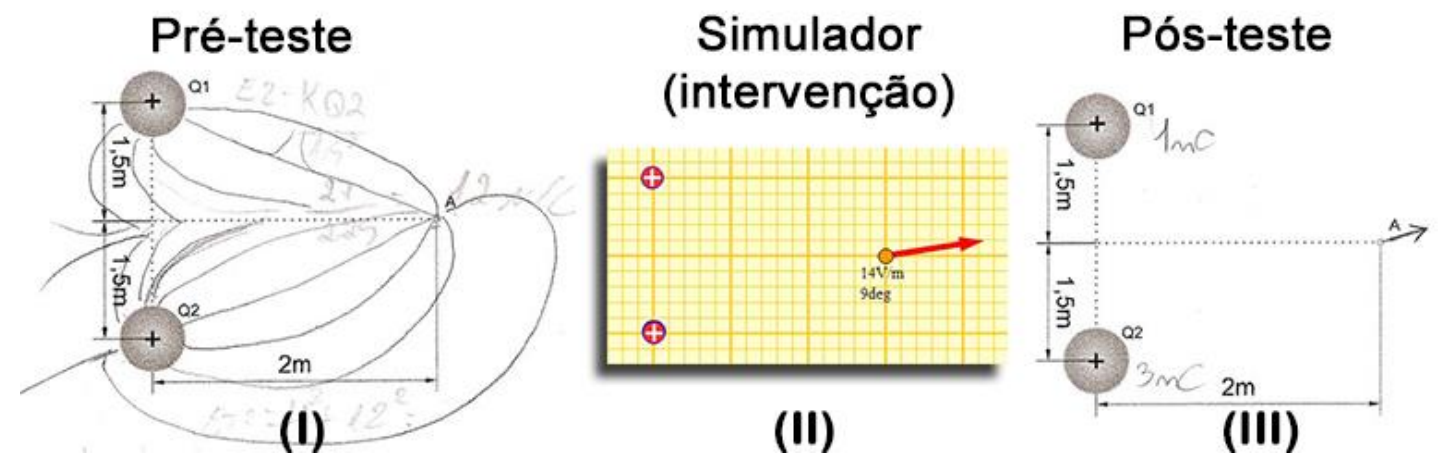

Figura 4 - (I) Representação desenhada por EZ de vetor campo elétrico, (II) imagem capturada da simulação, (III) representação desenhada por EZ de vetor campo elétrico. 
Em relação ao discurso não verbal, há também evidências de uma evolução cognitiva. $O$ estudante "EZ" ao descrever o vetor campo elétrico no espaço, resultante em função de duas cargas elétricas utiliza gestos não antes observados, para realizar estes gestos, ele necessita desenvolver novos drivers que são diferentes dos necessários para a visualização e que aparentemente ele não tinha, mas que desenvolveu a partir da interação com o software (mecanismo de processamento externo). Estes drivers foram integrados na estrutura cognitiva do estudante, pois mesmo sem a conexão com o computador, ele os utiliza e isso fica evidenciado na sequência da (Figura 5) e sua representação na (Figura 6).
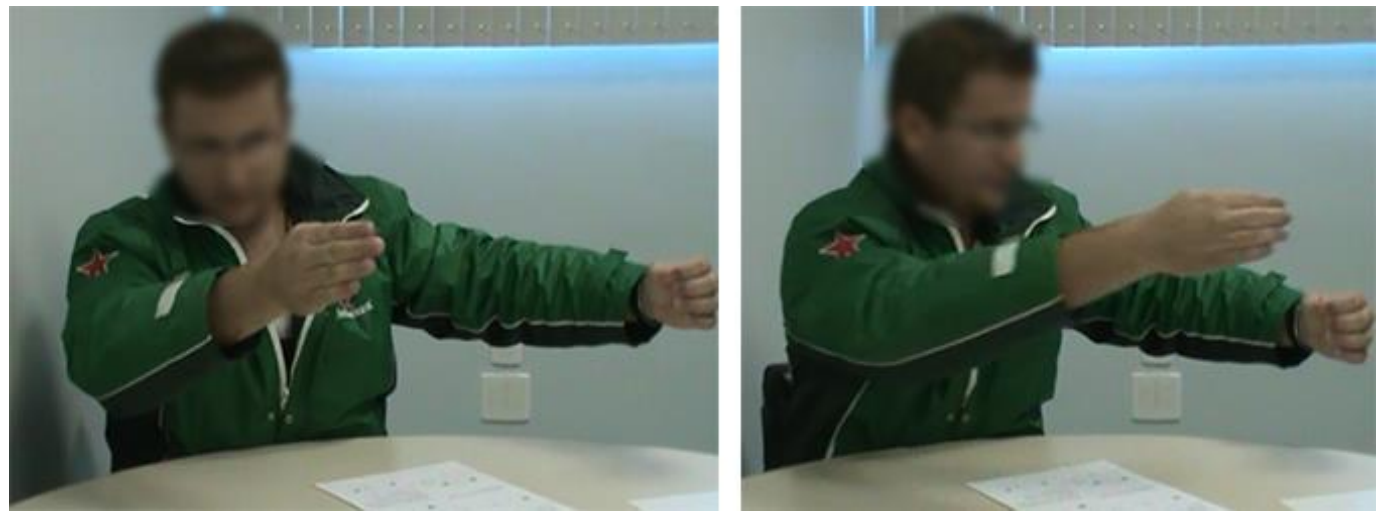

Figura 5 - Gestos do estudante EZ descrevendo “desvio" do vetor campo elétrico.

Estudante EZ: Daria para imaginar de novo com a mesa, duas mesas dai o ponto estivesse no meio das mesas, aqui é o positivo e tá passando para lá, a força está indo para lá assim [faz um movimento de afastamento criando uma linha imaginária], aqui tem o positivo então ela esta expelindo [abre e fecha as mãos repetidamente] só que aquele 1 ata negativa aquela mesa lá então o negativo esta puxando [faz um movimento de aproximação a um ponto imaginário] então a força do B esta indo até lá desculpa do positivo, esta indo até lá mas esta numa curva indo para o positivo [faz um movimento de curva]

\section{Pré-teste}

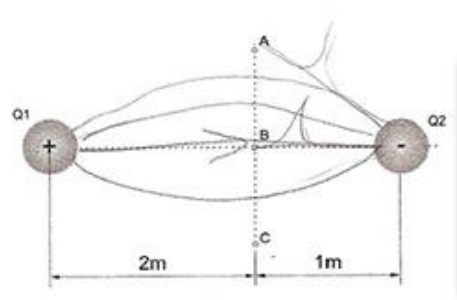

(1)
Simulador

(Intervenção)

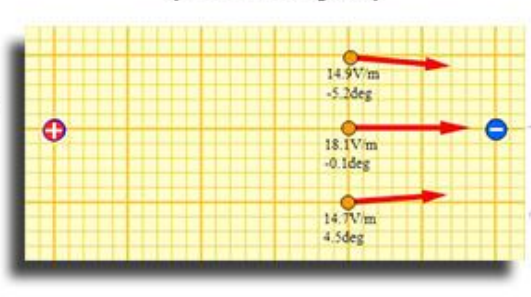

(II)

\section{Pós-teste}

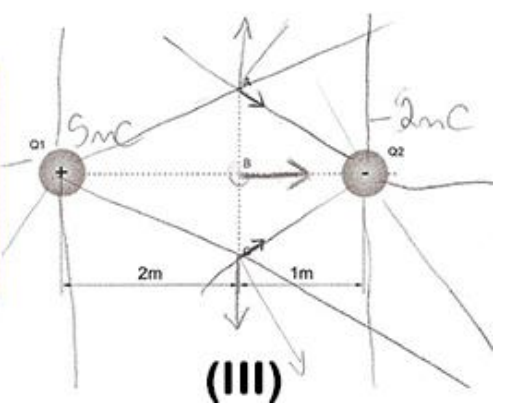

Figura 6 - (I) Representação desenhada por EZ de vetor campo elétrico, (II) imagem capturada da simulação, (III) representação desenhada por EZ de vetor campo elétrico.

Com o cruzamento dos resultados entendemos que a mediação com o software de simulação atua como um mecanismo de processamento externo, fazendo com que o estudante não apenas tivesse um melhor refinamento de seus drivers existentes como também possibilitou a criação de novos drivers. Drivers esses que são utilizados pelo estudante para resolução de situações problema, mesmo quando o contato com o mecanismo de processamento externo é eliminado. $\mathrm{O}$ diálogo acima evidencia uma das 
premissas da TMC: que o estudante, ao interagir com o mecanismo de processamento externo, cria, internamente, mecanismos análogos ao funcionamento do mecanismo externo, no caso, o software de simulação de campo elétrico.

Observe que para realizar a tarefa solicitada, no pré-teste, ao utilizar drivers psicofísicos adquiridos da física do experimento de limalha de ferro e ímã e do gerador de Van der Graaff, o estudante na realidade apenas retrata as linhas de campo elétrico nos pontos em torno das cargas (Figuras 3 e 5) ou até mesmo considerando um dos pontos (A) como gerando linhas de campo (Figura 2).

Estudante EZ: Então, é tanto carga negativa como positiva, então a positiva repele no caso que eu dei o exemplo anterior e a carga negativa atrai, positivo é como o exemplo do Van der Graaff onde a gente colocou os papeizinhos no momento que ligou ela repeliu [abre e fecha as mãos repetidamente], isso para mim é uma carga positiva e atrair seria... um exemplo que a gente teve foi colocar um imã em baixo e despedaçamos Bombril e eles ficaram alinhadinhos, mas ele ficou ali não repeliu, ai eu fiz o desenho como nos outros positivo ele repele e negativo ele atrai.

Já durante o pós-teste, o estudante trata estes pontos como cargas de prova (ou sensor de campo), que não são capazes de gerar campo elétrico, devido à aquisição de drivers hiperculturais provenientes da simulação (Figura 1). Isto, para nós, constitui evidência da aquisição de novos drivers que possibilitam-no avançar em termos de pensamento lógico, executando corretamente a tarefa solicitada.

Estudante EZ: (...) ai para desenhar o que eu imaginei aquele exercício que a gente fez no computador, então aqui esta mais forte, esquecendo isso aqui se fosse só usar isso aqui ela ficaria numa linha reta o campo de força, se fosse esquecer essa aqui ela ficaria para cá numa linha reta se as duas fossem da mesma força né [com o dedo descreve linhas no papel].

(...) mais a força deles esta menor que nem o desenho que a gente fazia com dois pontos, então acaba quanto mais distante menor a força em relação ao ponto, então o B tá na linha reta ele quase triplicou o valor, alias.... ele triplicou porque aqui tá... então o vetor no ponto B ele esta praticamente três vezes maior que no $\mathrm{A}$ e no $\mathrm{C}$, por isso que tentei representar a flechinha um pouco maior aqui.

\section{Considerações Finais}

Iniciamos esse trabalho com o objetivo de investigar se o uso de softwares de simulação auxiliaria o aprendizado de conceitos de campo elétrico. Quando em contato com esse mecanismo de processamento externo (software de simulação) parte de seu conteúdo é internalizado e essas novas informações confrontadas com os drivers existentes na estrutura cognitiva do estudante, a partir desse momento novos drivers são criados. E dessa forma que os drivers, que funcionam com interfaces, são aparentemente consolidados como scripts, da mesma forma que novos driver também são criados devido a essa interação hipercultural.

Assim que esses scripts são gerados, os estudantes liberam memória de trabalho que ampliam sua capacidade cognitiva, permitindo domínio progressivo de situaçõesproblemas e crescimento conceitual, como pode ser lido pela Teoria de Campos Conceituais. Este processo ocorre naturalmente, como percebemos nos registros do estudo exploratório.

A nosso ver, essa é uma das grandes perspectivas oferecidas pela hipercultura no aprendizado de conceitos científicos, além desta condição, alguns fatores podem contribuir também para o processo: a existência de uma didática centrada na resolução 
de problemas e a escolha de situações adequadas que possam desencadear o processo de conflito cognitivo capaz de mobilizar os estudantes ao aprendizado.

\section{Notas de Texto}

${ }^{1}$ Disponibilizado em: http://www.4shared.com/zip/77ii-aLt/Experimento.html

${ }^{2}$ Disponibilizado em: http://www.4shared.com/zip/77ii-aLt/Experimento.html

${ }^{3}$ Disponibilizado em: http://phet.colorado.edu/pt_BR/simulation/charges-and-fields

${ }^{4}$ Disponibilizado em: http://www.4shared.com/zip/77ii-aLt/Experimento.html

\section{Referências Bibliográficas}

MARTIN, José; SOLBES, Jordi. Diseño y evoluación de una propuesta para la enseñanza del concepto de campo en Física. Enseñanza de las ciencias, v. 19, n. 3, p. 393-403, 2001.

MONAGHAN, J.M.; CLEMENT, J. Use of a computer simulation to develop mental simulations for understanding relative motion concepts. International Journal of Science Education., v. 21, n. 9, p. 921- 944, 1999

SCHERR, R. Gesture analysis for physics education researchers. Physical Review Special Topics - Physics Education Research, v. 4, n 010101, 2008.

SOUZA, B. C. DE. A Teoria da Mediação Cognitiva: Os impactos cognitivos da Hipercultura e da Mediação Digital, 2004. Universidade Federal de Pernambuco. Centro de Filosofia e Ciências Humanas. Disponível em:

$<$ http://www.liber.ufpe.br/teses/arquivo/20040617095205.pdf>. Acesso em: 15 jan. 2013

SOUZA, B. C.; SILVA, A. S.; SILVA, A. M.; ROAZZI, A.; SILVA, S. L. C. Putting the Cognitive Mediation Networks Theory to the test: Evaluation of a framework for understanding the digital age. Computers in Human Behavior, v. 28, n. 6, p. 23202330, 2012. Elsevier Ltd. Disponível em:

<http://linkinghub.elsevier.com/retrieve/pii/S0747563212002026>. Acesso em: 15 jan. 2013.

STEPHENS, A. LYNN; CLEMENT, JOHN J. Documenting the use of expert scientific reasoning processes by high school physics students. Physical Review Special Topics - Physics Education Research, 2010.

VERGNAUD, G. Quelques orientations théoriques et methodologiques dês recherches françaises en didactique des mathématiques. Recherches en Didactique des Mathématiques, v.2, n.2, p. 215-232, 1981.

Teoria dos campos conceituais. In: NASSER, L. (Ed.). Anais do $1^{\circ}$ Seminário Internacional de Educação Matemática. Rio de Janeiro, p. 1-26, 1993.

VYGOTSKY, L. S. A formação Social da Mente. São Paulo, SP: Martins Fontes Editora LTDA, 1984. 